\title{
Spectroscopic versus evolutionary masses
}

\author{
Artemio Herrero \\ Instituto de Astrofisica de Canarias, \\ C/ Via Lactea s/n, E-38200, La Laguna, Tenerife, Spain \\ email: ahd@iac.es
}

\begin{abstract}
We describe the present status of the mass discrepancy in the Upper HR Diagram.
\end{abstract}
Keywords. stars: fundamental parameters (masses)

The mass determination of isolated stars is particularly difficult in the Upper HR Diagram, and yet it is a fundamental parameter for our understanding of the stellar structure and evolution and the interpretation of integrated spectra, stellar populations and the IMF. The two main methods are both indirect: analysis of the stellar spectra by means of model atmospheres (spectroscopic masses, SM) and comparison with evolutionary tracks, after placing the star on a HRD (evolutionary masses, EM). Herrero et al. (1992) noted that for $\mathrm{O}$ stars these two methods gave different results, and called this situation the mass discrepancy, with the EM being systematically larger than the SM.

Model improvements during the next decade, including line-blanketing and mass-loss led to new, higher SM (due to the influence of mass-loss) and lower EM (due to lower effective temperatures and luminosities). These changes solved most of the mass discrepancy (see Repolust et al. 2004). Other recent analyses confirmed that both masses now agree within the limits of the error bars. However, second order effects might still be present: $(a)$ for SM between $20-60 \mathrm{M}_{\odot}$ there might be a milder form of the mass discrepancy, with EM being still about $30 \%$ larger; (b) for SM below $20 \mathrm{M}_{\odot}$, some discrepancy may still be present; and $(c)$ for $\mathrm{SM}$ above $60 \mathrm{M}_{\odot}$ an inverted form of the mass discrepancy (with SM larger than EM) may appear. These results are consistent with analyses of $\mathrm{O}$ and $\mathrm{B}$ stars in the Magellanic Clouds and the Milky Way by different authors.

Mokiem (2006) has presented a plot of the mass discrepancy versus the stellar helium abundance. Their findings (He-enriched O-supergiants, show no mass discrepancy; Heenriched O-dwarfs show mass discrepancy) are consistent with the analyses of Herrero \& Lennon (2004), who proposed that the more massive stars have an evolution dominated by mass-loss and the intermediate massive stars have an evolution dominated by rotation.

In conclusion, there is no clear supporting evidence for a general mass-discrepancy problem. We find good agreement between EM and SM in most cases, (and with dynamical masses when comparing with binary systems). Second order effects may still be present. More sophisticated model atmospheres (clumping, 2-D effects) and more homogeneous evolutionary tracks at different $Z$ are needed for the comparisons.

\section{References}

Herrero, A., Kudritzki, R.-P., Vilchez, J. M., et al. 1992, A\&GA, 261, 209

Herrero, A., \& Lennon, D. J. 2004, in: A. Maeder \& P. Eenens (eds.), Steller Rotation, Proc. IAU Symp. No. 215 (San Francisco: ASP), p. 209

Mokiem, R. M. 2006, PhD thesis, University of Amsterdam

Repolust, T., Puls, J., \& Herrero, A. 2004, A\& $A$, 415, 349 